\title{
BOUNDS AND A MAJORIZATION FOR THE REAL PARTS OF THE ZEROS OF POLYNOMIALS
}

\author{
FUAD KITTANEH
}

(Communicated by Joseph A. Ball)

\begin{abstract}
We apply some eigenvalue inequalities to the real parts of the Frobenius companion matrices of monic polynomials to establish new bounds and a majorization for the real parts of the zeros of these polynomials.
\end{abstract}

\section{INTRODUCTION}

Matrix analysis methods have been used by several mathematicians to obtain new proofs of classical bounds for the zeros of polynomials and to derive new bounds for these zeros. These methods include eigenvalue locations, matrix norms computations, eigenvalue-singular value majorization relations, and numerical radii estimations. See, e.g., [1]-2], 4]-7], [9]-[14], 16], and the references therein.

Let

$$
p(z)=z^{n}+a_{n} z^{n-1}+\cdots+a_{2} z+a_{1}
$$

be a monic polynomial of degree $n \geq 2$, with complex coefficients.

Then the Frobenius companion matrix of $p$ is given by

$$
C(p)=\left[\begin{array}{ccccc}
-a_{n} & -a_{n-1} & \cdots & -a_{2} & -a_{1} \\
1 & 0 & \cdots & 0 & 0 \\
0 & 1 & \cdots & 0 & 0 \\
\vdots & \vdots & \ddots & \vdots & \vdots \\
0 & 0 & \cdots & 1 & 0
\end{array}\right] .
$$

It is well known that the zeros of $p$ are exactly the eigenvalues of $C(p)$. See, e.g., [7. p. 316].

Using a numerical radius estimation of $C(p)$, it has been shown in [5] (see also 11] for a different proof) that if $z$ is any zero of $p$, then

$$
|z| \leq \cos \frac{\pi}{n+1}+\frac{1}{2}\left(\left|a_{n}\right|+\sqrt{\sum_{j=1}^{n}\left|a_{j}\right|^{2}}\right) .
$$

Received by the editors August 9, 2004 and, in revised form, September 28, 2005.

2000 Mathematics Subject Classification. Primary 15A18, 15A42, 26C10, $30 \mathrm{C} 15$.

Key words and phrases. Frobenius companion matrix, zeros of polynomials, eigenvalue, majorization.

(C)2006 American Mathematical Society Reverts to public domain 28 years from publication 
In this paper we apply some eigenvalue inequalities to the real part of $C(p)$ to establish new bounds and a majorization for the real parts of the zeros of $p$ that are related to the bound (3).

\section{Preliminary Results}

Let $z_{1}, z_{2}, \cdots, z_{n}$ be the zeros of $p$ (or the eigenvalues of $C(p)$ ). To obtain our new bounds and majorization for $\operatorname{Re} z_{1}, \cdots, \operatorname{Re} z_{n}$, we need several lemmas involving inequalities and majorization relations for eigenvalues.

Let $M_{n}(\mathbb{C})$ denote the algebra of all $n \times n$ complex matrices. For $A \in M_{n}(\mathbb{C})$, the eigenvalues of $A$ are denoted by $\lambda_{1}(A), \lambda_{2}(A), \cdots, \lambda_{n}(A)$. If $A$ is Hermitian, then the eigenvalues of $A$ are arranged in such a way that $\lambda_{1}(A) \geq \lambda_{2}(A) \geq \cdots \geq \lambda_{n}(A)$.

For two sequences of real numbers arranged in decreasing order,

$$
x=\left(x_{1}, x_{2}, \cdots, x_{n}\right) \text { and } y=\left(y_{1}, y_{2}, \cdots, y_{n}\right),
$$

we say that $x$ is majorized by $y$ if

$$
\sum_{j=1}^{k} x_{j} \leq \sum_{j=1}^{k} y_{j} \quad \text { for } \quad k=1,2, \cdots, n-1
$$

and

$$
\sum_{j=1}^{n} x_{j}=\sum_{j=1}^{n} y_{j}
$$

For the theory of majorization, the reader is referred to [3], $[8]$, and $[15]$.

Lemma 1. Let $A \in M_{n}(\mathbb{C})$ with real part $\operatorname{Re} A=\frac{A+A^{*}}{2}$. Then

$$
\lambda_{n}(\operatorname{Re} A) \leq \operatorname{Re} \lambda_{j}(A) \leq \lambda_{1}(\operatorname{Re} A) \quad \text { for } \quad j=1,2, \cdots, n .
$$

Lemma 2. Let $B, C \in M_{n}(\mathbb{C})$ be Hermitian. Then

$$
\lambda_{j}(B)+\lambda_{n}(C) \leq \lambda_{j}(B+C) \leq \lambda_{j}(B)+\lambda_{1}(C) \quad \text { for } \quad j=1,2, \cdots, n .
$$

In particular,

$$
\lambda_{1}(B+C) \leq \lambda_{1}(B)+\lambda_{1}(C)
$$

and

$$
\lambda_{n}(B)+\lambda_{n}(C) \leq \lambda_{n}(B+C) .
$$

Lemma 3. Let $A \in M_{n}(\mathbb{C})$ with eigenvalues arranged in such a way that $\operatorname{Re} \lambda_{1}(A) \geq$ $\operatorname{Re} \lambda_{2}(A) \geq \cdots \geq \operatorname{Re} \lambda_{n}(A)$. Then

$$
\sum_{j=1}^{k} \operatorname{Re} \lambda_{j}(A) \leq \sum_{j=1}^{k} \lambda_{j}(\operatorname{Re} A) \quad \text { for } \quad k=1,2, \cdots, n-1
$$

and

$$
\sum_{j=1}^{n} \operatorname{Re} \lambda_{j}(A)=\sum_{j=1}^{n} \lambda_{j}(\operatorname{Re} A)
$$


Lemma 4. Let $B, C \in M_{n}(\mathbb{C})$ be Hermitian. Then

$$
\sum_{j=1}^{k} \lambda_{j}(B+C) \leq \sum_{j=1}^{k} \lambda_{j}(B)+\sum_{j=1}^{k} \lambda_{j}(C) \quad \text { for } \quad k=1,2, \cdots, n-1
$$

and

$$
\sum_{j=1}^{n} \lambda_{j}(B+C)=\sum_{j=1}^{n} \lambda_{j}(B)+\sum_{j=1}^{n} \lambda_{j}(C) .
$$

Lemmas 1 and 2 are immediate consequences of the Courant-Fischer-Weyl minimax principle, and Lemmas 3 and 4 follow from Ky Fan's maximum principle. See, e.g., [3, Chapter III], [7, Chapter 4], [8, Chapter 3], and [15, Chapter 9]. It should be mentioned here that the real parts in Lemmas 1 and 3 can be replaced by the imaginary parts.

\section{MAin RESUlts}

Our new bounds for the real parts of the zeros of $p$ can be stated as follows.

Theorem 1. For $j=1,2, \cdots, n$, we have

$$
\begin{gathered}
\frac{1}{2}\left(-\operatorname{Re} a_{n}-\sqrt{\left(\operatorname{Re} a_{n}\right)^{2}+\sum_{j=1}^{n-1}\left|a_{j}\right|^{2}}\right)+\cos \frac{n \pi}{n+1} \leq \operatorname{Re} z_{j} \\
\leq \frac{1}{2}\left(-\operatorname{Re} a_{n}+\sqrt{\left(\operatorname{Re} a_{n}\right)^{2}+\sum_{j=1}^{n-1}\left|a_{j}\right|^{2}}\right)+\cos \frac{\pi}{n+1} .
\end{gathered}
$$

Proof. It follows from (2) that

$$
\operatorname{Re} C(p)=\left[\begin{array}{ccccc}
-\operatorname{Re} a_{n} & \left(1-a_{n-1}\right) / 2 & -a_{n-2} / 2 & \cdots & -a_{1} / 2 \\
\left(1-\bar{a}_{n-1}\right) / 2 & 0 & 1 / 2 & \cdots & 0 \\
-\bar{a}_{n-2} / 2 & 1 / 2 & 0 & \ddots & 0 \\
\vdots & \vdots & \vdots & & 1 / 2 \\
& & & \ddots & \\
-\bar{a}_{1 / 2} & 0 & 0 \cdots & 1 / 2 & 0
\end{array}\right] .
$$

Thus,

$$
\operatorname{Re} C(p)=S_{n}+T_{n}
$$

where $S_{n}$ is the partitioned matrix

$$
S_{n}=\left[\begin{array}{cc}
-\operatorname{Re} a_{n} & x^{*} \\
x & 0
\end{array}\right]
$$

with $x=\left[-\frac{1}{2} \bar{a}_{n-1},-\frac{1}{2} \bar{a}_{n-2}, \cdots,-\frac{1}{2} \bar{a}_{1}\right]^{t}$, and $T_{n}$ is the $n \times n$ tridiagonal matrix

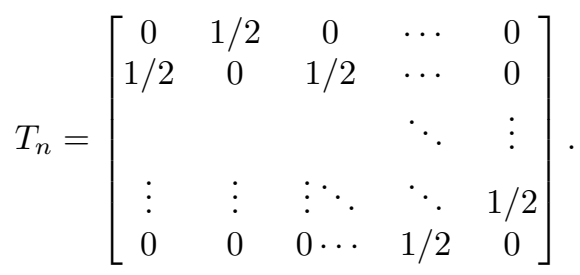


It can easily be shown that the eigenvalues of $S_{n}$ are

$$
\begin{aligned}
& \lambda_{1}\left(S_{n}\right)=\frac{1}{2}\left(-\operatorname{Re} a_{n}+\sqrt{\left(\operatorname{Re} a_{n}\right)^{2}+\sum_{j=1}^{n-1}\left|a_{j}\right|^{2}}\right), \\
& \lambda_{n}\left(S_{n}\right)=\frac{1}{2}\left(-\operatorname{Re} a_{n}-\sqrt{\left(\operatorname{Re} a_{n}\right)^{2}+\sum_{j=1}^{n-1}\left|a_{j}\right|^{2}}\right),
\end{aligned}
$$

and

$$
\lambda_{j}\left(S_{n}\right)=0 \quad \text { for } \quad j=2, \cdots, n-1 .
$$

It is well known that the eigenvalues of $T_{n}$ are

$$
\lambda_{j}\left(T_{n}\right)=\cos \frac{j \pi}{n+1} \quad \text { for } \quad j=1,2, \cdots, n .
$$

Applying Lemmas 1 and 2 to $C(p)$ and the Hermitian matrices $S_{n}, T_{n}$, respectively, we obtain

$$
\lambda_{n}\left(S_{n}\right)+\lambda_{n}\left(T_{n}\right) \leq \lambda_{n}(\operatorname{Re} C(p)) \leq \operatorname{Re} z_{j} \leq \lambda_{1}(\operatorname{Re} C(p)) \leq \lambda_{1}\left(S_{n}\right)+\lambda_{1}\left(T_{n}\right)
$$

for $j=1,2, \cdots, n$. Now, the desired bounds (12) follow from (13), (14), (16), and (17).

By considering the imaginary part of $C(p)$, an analysis similar to that used in deriving the bounds (12) enables us to obtain analogous bounds for the imaginary parts of the zeros of $p$. These bounds, when combined with (12), describe a rectangle that contains all the zeros of $p$. Related rectangles have been described in [11, Theorem 3].

Our second result gives a majorization for the real parts of the zeros of $p$.

Theorem 2. If the zeros of $p$ are arranged in such a way that $\operatorname{Re} z_{1} \geq \operatorname{Re} z_{2} \geq$ $\cdots \geq \operatorname{Re} z_{n}$, then

(18) $\sum_{j=1}^{k} \operatorname{Re} z_{j} \leq \frac{1}{2}\left(-\operatorname{Re} a_{n}+\sqrt{\left(\operatorname{Re} a_{n}\right)^{2}+\sum_{j=1}^{n-1}\left|a_{j}\right|^{2}}\right)+\frac{1}{2}\left(\frac{\sin \left(\frac{2 k+1}{2 n+2}\right) \pi}{\sin \frac{\pi}{2 n+2}}\right)-\frac{1}{2}$

for $k=1,2, \cdots, n-1$ and

$$
\sum_{j=1}^{n} \operatorname{Re} z_{j}=-\operatorname{Re} a_{n}
$$


Proof. Applying Lemmas 3 and 4 to $C(p)$ and the Hermitian matrices $S_{n}, T_{n}$, respectively, we obtain, in view of our analysis in the proof of Theorem 1, that

$$
\begin{aligned}
\sum_{j=1}^{k} \operatorname{Re} z_{j} & =\sum_{j=1}^{k} \operatorname{Re} \lambda_{j}(C(p)) \\
& \leq \sum_{j=1}^{k} \lambda_{j}(\operatorname{Re}(C(p))) \\
& \leq \sum_{j=1}^{k} \lambda_{j}\left(S_{n}\right)+\sum_{j=1}^{k} \lambda_{j}\left(T_{n}\right) \\
& =\frac{1}{2}\left(-\operatorname{Re} a_{n}+\sqrt{\left(\operatorname{Re} a_{n}\right)^{2}+\sum_{j=1}^{n-1}\left|a_{j}\right|^{2}}\right)+\sum_{j=1}^{k} \cos \frac{j \pi}{n+1}
\end{aligned}
$$

for $k=1,2, \cdots, n-1$. But, for every real number $t \neq 2 m \pi$ ( $m$ is an integer), we have

$$
\sum_{j=1}^{k} \cos j t=\frac{1}{2}\left(\frac{\sin (2 k+1) \frac{t}{2}}{\sin \frac{t}{2}}\right)-\frac{1}{2} .
$$

Thus,

$$
\sum_{j=1}^{k} \cos \frac{j \pi}{n+1}=\frac{1}{2}\left(\frac{\sin \left(\frac{2 k+1}{2 n+2}\right) \pi}{\sin \frac{\pi}{2 n+2}}\right)-\frac{1}{2},
$$

which, together with (20), yields the desired majorization (18). The relation (19) follows from the fact that $\sum_{j=1}^{n} z_{j}=-a_{n}$. This completes the proof of the theorem.

Finally, we remark that by similar arguments, we can establish analogous majorization for the imaginary parts of the zeros of $p$. It should be mentioned here that inequalities for the absolute values of the imaginary parts of the zeros of polynomials have been given in [16].

\section{REFERENCES}

[1] A. A. Abdurakhmanov, The geometry of the Hausdorff domain in localization problems for the spectrum of arbitrary matrices, Math. USSR-Sb. 59 (1988), 39-51. MR0868600 (88e:47010)

[2] Y. A. Alpin, M.-T. Chien and L. Yeh, The numerical radius and bounds for zeros of a polynomial, Proc. Amer. Math. Soc. 131 (2003), 725-730. MR.1937409(2003h:26021)

[3] R. Bhatia, Matrix Analysis, Springer, New York, 1997. MR.1477662 (98i:15003)

[4] M. Fujii and F. Kubo, Operator norms as bounds for roots of algebraic equations, Proc. Japan Acad. Sci. 49 (1973), 805-808. MR0364310 (51:565)

[5] M. Fujii and F. Kubo, Buzano's inequality and bounds for roots of algebraic equations, Proc. Amer. Math. Soc. 117 (1993), 359-361. MR1088441 (93d:47014)

[6] K. E. Gustafson and D. K. M. Rao, Numerical Range, Springer, New York, 1997. MR.1417493 (98b:47008)

[7] R. A. Horn and C. R. Johnson, Matrix Analysis, Cambridge Univ. Press, Cambridge, 1985. MR0832183 (87e:15001)

[8] R. A. Horn and C. R. Johnson, Topics in Matrix Analysis, Cambridge Univ. Press, Cambridge, 1991. MR1091716 (92e:15003) 
[9] F. Kittaneh, Singular values of companion matrices and bounds on zeros of polynomials, SIAM J. Matrix Anal. Appl. 16 (1995), 333-340. MR1311437 (95m:15015)

[10] F. Kittaneh, A numerical radius inequality and an estimate for the numerical radius of the Frobenius companion matrix, Studia Math. 158 (2003), 11-17. MR2014548 (2004i:15022)

[11] F. Kittaneh, Bounds for the zeros of polynomials from matrix inequalities, Arch. Math. (Basel) 81 (2003), 601-608. MR2029723 (2004j:15035)

[12] H. Linden, Bounds for the zeros of polynomials from eigenvalues and singular values of some companion matrices, Linear Algebra Appl. 271 (1998), 41-82. MR:1485162 (98m:65059)

[13] H. Linden, Numerical radii of some companion matrices and bounds for the zeros of polynomials, in: Analytic and Geometric Inequalities and Applications, Math. Appl. 478, Kluwer, Dordrecht, 1999, 205-229. MR1785871 (2001i:15031)

[14] M. Marden, Geometry of Polynomials, 2nd ed., Math. Surveys 3, Amer. Math. Soc., Providence, RI, 1966. MR0225972 (37:1562)

[15] A. W. Marshall and I. Olkin, Inequalities: Theory of Majorization and Its Applications, Academic Press, New York, 1979. MR 0552278 (81b:00002)

[16] G. Schmeisser, Sharp inequalities for the zeros of polynomials and power series, Result. Math. 39 (2001), 333-344. MR1834579 (2002c:30011)

Department of Mathematics, University of Jordan, Amman, Jordan

E-mail address: fkitt@ju.edu.jo 